Stochastic Equilibrium Models for Generation Capacity Expansion

Andreas Ehrenmann and Yves Smeers

September 2010

CWPE 1041 \& EPRG 1019 


\title{
Stochastic Equilibrium Models for Generation Capacity Expansion
}

\author{
EPRG Working Paper 1019 \\ Cambridge Working Paper in Economics 1041
}

\section{Andreas Ehrenmann and Yves Smeers}

\section{Abstract}

Capacity expansion models in the power sector were among the first applications of operations research to the industry. We introduce stochastic equilibrium versions of these models that we believe provide a relevant context for looking at the current very risky market where the power industry invests and operates. We then look at the insertion of risk related investment practices that developed with the new environment and may not be easy to accommodate in an optimization context. Specifically we consider the use of plant specific discount rates that we derive by including stochastic discount rates in the equilibrium model. Linear discount factors only price systematic risk. We therefore complete the discussion by inserting different risk functions (for different agents) in order to account for additional unpriced idiosyncratic risk in investments. These different models can be cast in a single mathematical representation but they do not have the same mathematical properties. We illustrate the impact of these phenomena on a small but realistic example.

Keywords

JEL Classification

Contact

Publication

Financial Support capacity adequacy, risk functions, stochastic equilibrium models, stochastic discount factors

D58, D81, Q40,

andreas.ehrenmann@gdfsuez.com September 2010

GDF SUEZ
$\mathrm{E} \cdot \mathrm{S} \cdot \mathrm{R} \cdot \mathrm{C}$ ECONOMIC $\&$ SOCIAL RESEARCH COUNCIL 


\title{
Stochastic Equilibrium Models for Generation Capacity Expansion
}

\author{
Andreas Ehrenmann* and Yves Smeers ${ }^{\dagger}$
}

September 2010

\begin{abstract}
Capacity expansion models in the power sector were among the first applications of operations research to the industry. The models lost some of their appeal at the inception of restructuring even though they still offer a lot of possibilities and are in many respect irreplaceable provided they are adapted to the new environment. We introduce stochastic equilibrium versions of these models that we believe provide a relevant context for looking at the current very risky market where the power industry invests and operates. We then take up different questions raised by the new environment. Some are due to developments of the industry like demand side management: an optimization framework has difficulties accommodating them but the more general equilibrium paradigm offers additional possibilities. We then look at the insertion of risk related investment practices that developed with the new environment and may not be easy to accommodate in an optimization context. Specifically we consider the use of plant specific discount rates that we derive by including stochastic discount rates in the equilibrium model. Linear discount factors only price systematic risk. We therefore complete the discussion by inserting different risk functions (for different agents) in order to account for additional unpriced idiosyncratic risk in investments. These different models can be cast in a single mathematical representation but they do not have the same mathematical properties. We illustrate the impact of these phenomena on a small but realistic example.
\end{abstract}

Key words: capacity adequacy, risk functions, stochastic equilibrium models, stochastic discount factors

* GDF SUEZ, Center of Expertise in Economic Modeling and Studies Disclaimer: The views and opinions expressed herein do not necessarily state or reflect those of GDF Suez

$\dagger^{\dagger}$ School of engineering and CORE, Université catholique de Louvain, Belgium 


\section{Introduction}

The restructuring of the electricity industry led to an explosion of literature transposing and extending optimization models of short-term operations to equilibrium models of electricity markets with given infrastructure. The optimal dispatch and its extension to the optimal power flow are the reference optimization paradigms at the origin of that literature. They cover energy and transmission and sometimes encompass other services such as reserve. These models were instrumental in analyzing market design. Variations of these models that encompass market power were also extensively developed to examine market structure.

Capacity expansion models are as old as the optimal dispatch models but the transition from optimization to equilibrium models has not yet taken place. The early optimization models of capacity expansion go back to the late fifties when the industry was still regulated (Morlat and Bessière, 1971). The problem was first formulated as a linear program but further developments quickly followed suit and extensions covered all types of optimization techniques. Capacity expansion, which was initially seen as a true planning exercise was easily reinterpreted in terms of equilibrium in a competitive energy economy in the early seventies after the first energy crisis. The power industry of the seventies was still regulated on a cost plus basis that largely protected it from risk. Deterministic models were thus satisfactory in the situation of the time. Restructuring removed that protection at the same time that various new policies and external events dramatically increased the risk surrounding the electricity sector. This emergence of risk in the investment process strongly suggests to move the analysis from a deterministic to a stochastic environment. The question is thus to transpose former optimization capacity expansion models to stochastic equilibrium models. This extension is the subject of this paper.

The first analysis of a capacity expansion problem in terms of a stochastic equilibrium capacity expansion model in the energy area is probably found in Haurie et al. (1988). The model deals with gas developments and was formulated as an open loop Cournot equilibrium under demand uncertainty. This model could be converted to an optimization model that was later used in Gürkan et al. (1999) to illustrate the method of "Sample Path" since elaborated by several authors. Lin and Fukushima (2009) recently reviewed different models of stochastic equilibrium, among them the one used by Gürkan et al. (1999) in their application of sample path to the investments in gas production. This model is stated as a stochastic variational inequality problem; we adopt the closely related formulation of stochastic com- 
plementarity problems as the modeling paradigm of the investment problem throughout this paper.

Section 1 of the paper introduces a very simple and standard two-stage version of a stochastic optimization capacity expansion model as could have been constructed in the regulated environment. We adopt a standard stochastic programming approach and present the model in terms of its first and second stages. We then immediately reformulate this problem in the stochastic equilibrium format that drives the whole paper. Section 2 discusses the possibilities and limitations of stochastic equilibrium models to account for idiosyncrasies of restructured electricity markets.

The rest of the paper analyses different risk issues encountered in the investment process. The standard approach in investment problems is to reflect risk in the discount rate. The discount rate is normally regulated when the industry operates as a monopoly; this may have raised economic controversies but did not create modeling difficulties as the discount rate is just a single parameter of the model. The problem is quite different in a world where "project finance" drives the capacity expansion process and requires that plants are evaluated on the basis of different discount rates. The CAPM and the APT are the reference theories for finding these discount rates. Expositions of these theories can be found in any textbook of corporate finance and we take them for granted. The adoption of a project finance approach therefore requires the stochastic equilibrium model to accommodate plant specific discount rates while maintaining the interpretation of a competitive economy that is the justification of the model. A first treatment of the question is given in Section 3 leading to a fixed point formulation. Section 4 adopts an alternative, probably more rigorous but also less usual representation of risk. Starting again from a CAPM based formulation it assumes that the different risks affecting plants can be taken care of by modifying the payoff of the different plants using a linear stochastic discount rate. Discounting is then conducted at the risk free rate but with risk adjusted cash flows computed with CAPM based stochastic discount rates. Section 5 considers an alternative version of the risk neutral discounting where the adjustment to the cash flow is derived from risk functions. Risk functions were initially developed by Artzner et al. (1999) and have been recently cast in an optimization context (see the book by Shapiro et al., 2009 for a comprehensive treatment). We extend this view to an equilibrium context to construct alternative adjustments of the cash flows of the plants. We provide a simplified but realistic illustration of these notions in Section 6. Conclusion summarizes the paper. In order to simplify the presentation the discussion is entirely conducted on a two or three stages models depending of our needs. 


\section{The basic capacity expansion model}

\subsection{The optimization model}

Consider a two-stage set up where one invests in a mix of new technologies in stage 0 and operates them in stage 1 . The objective is to satisfy a time segmented, price insensitive demand so as to minimize total (annual in this simple case) cost. The first versions of these models go back to the late fifties. They were initially formulated as linear programs and later expanded to take advantage of essentially all optimization techniques. We introduce these models as follows.

Consider a set of capacity types $K$ and a load duration curve decomposed in different time segments $L$ as depicted in Figure 1. The left figure gives a general decomposition and characterizes each time segment by its duration $\tau(\ell)$ and demand level $d(\ell)$. The right figure depicts the particular case of a decomposition into peak and off peak segments.
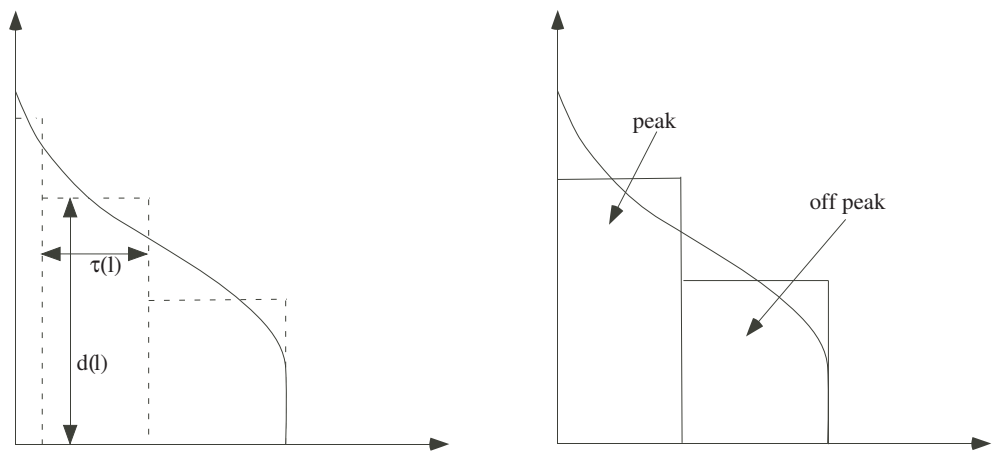

Figure 1: Decomposition of the load duration curve

Assume in order to simplify the presentation that there is no existing capacity. We introduce the following notation: $x(k)$ is the investment in capacity (in $\mathrm{MW}$ ) of technology $k \in K$ in the period; this capacity is operated at level $y(k, \ell)$ (in MW) in time segment $\ell$ of duration $\tau(\ell$ ) (in hours) when demand level is $d(\ell)$ (in MW). $\sum_{\ell} \tau(\ell)=8760$ is the number of hours in a year. The annual investment cost of technology $k$ is $I(k)$ (in $€ / \mathrm{MW}$ ) and its (constant) marginal operating cost is $c(k)$ (in $\in / \mathrm{Mwh}$ ). $P C$ is the Value of Lost load (VOLL), that is the economic value (in $€ / \mathrm{Mwh}$ ) of unsatisfied electricity demand. The notion has been around since several decades but has so far escaped any precise evaluation. VOLL is 
thus often taken as a conventional value ranging between 1000 and $10000 € /$ Mwh. PC can alternatively be interpreted as a price cap, that is an upper bound on the electricity price set by the Regulator (figures ranging between 300 and $3000 € /$ Mwh are then found in practice); $z(\ell)$ is the unsatisfied demand in time segment $\ell$ (in MW).

Adopting the standard two stage approach of stochastic programming, we successively write the second and first stage optimization problems (dual variables are stated at the right of the equations) as follows. The (short term) operations problem is stated as the following short term cost minimization problem

$$
Q(x) \equiv \min _{y, z} \sum_{\ell \in L} \tau(\ell)\left[\sum_{k \in K} c(k) y(k, \ell)+P C z(\ell)\right]
$$

s.t.

$$
\begin{array}{cc}
0 \leq x(k)-y(k, \ell) & \tau(\ell) \mu(k, \ell) \\
0 \leq \sum_{k \in K} y(k, \ell)+z(\ell)-d(\ell) & \tau(\ell) \pi(\ell) \\
0 \leq y(k, \ell) . &
\end{array}
$$

The (long term) investment problem is stated as a long term cost minimization problem

$$
\min _{x \geq 0} \sum_{k \in K} I(k) x(k)+Q(x) .
$$

In accordance with the units used for defining the parameters and primal variables, $\mu(k, \ell)$ and $\pi(\ell)$ are in $€ / \mathrm{Mw}$. It is more convenient to refer to them as $€ / \mathrm{Mwh}$.

\subsection{The equilibrium version of the optimization model}

The conversion of the optimization model to an equilibrium model is obtained by writing duality relations. The KKT conditions of the operations problem are stated as

$$
\begin{aligned}
& 0 \leq x(k)-y(k, \ell) \perp \mu(k, \ell) \geq 0 \\
& 0 \leq \sum_{k \in K} y(k, \ell)+z(\ell)-d(\ell) \perp \pi(\ell) \geq 0 \\
& 0 \leq c(k)+\mu(k, \ell)-\pi(\ell) \perp y(k, \ell) \geq 0 \\
& 0 \leq P C-\pi(\ell) \perp z(\ell) \geq 0 .
\end{aligned}
$$


Those of the investment problem are

$$
0 \leq I(k)-\sum_{\ell \in L} \tau(\ell) \mu(k, \ell) \perp x(k) \geq 0 .
$$

Complementarity formulations of capacity expansion models have been used by Gürkan et al. (2009) to examine different investment incentive policies in restructured markets. Our focus in this paper is different; we assume a single investment incentive mechanism throughout the paper (as embedded in the model (6) to (10) and usually referred to as "energy only"); we then concentrate on the investment cost $I(k)$ that we examine through different theories of corporate finance.

Relations (6) to (10) can easily be interpreted in terms of a perfect competition equilibrium. For the sake of brevity, we present this discussion in Appendix 1 and only report here the economic interpretation of the dual variables that play a key role in the rest of the discussion. Most important for our purpose, $\mu(k, \ell)$ is the hourly marginal value of capacity $k$ in time segment $\ell$. It is zero if the capacity is not fully utilized in that time segment; it is positive and $\tau(\ell) \mu(k, \ell)$ measures the hourly marginal decrease of the operating cost of the system in time segment $\ell$ if one adds a unit of capacity $k$. The hourly marginal generation cost in time segment $\ell$ is measured by $\pi(\ell)$. It is the sum of the operating cost $c(k, \ell)$ and of the hourly marginal value $\mu(k, \ell)$ of capacity $k$ when it is operating. The price $\pi(\ell)$ is set to $P C$ when load is curtailed.

The discussion developed in this paper focuses on the investment criterion (10). Its interpretation is that one invests in technology $k$ when the investment cost $I(k)$ is equal to the weighted (by $\tau(\ell)$ ) sum of the hourly marginal values of the capacity $\mu(k, \ell)$ computed over all time segments. We refer to this weighted sum $\sum_{\ell} \tau(\ell) \mu(k, \ell)$ as the gross margin of plant $k$ and note it in abridged form $\mu(k) . \mu(K)$ is the vector of the $\mu(k)$ for $k \in K$. One does not invest in technology $k$ when the gross margin is insufficient to cover $I(k)$. Because of the interactions between the operation and investment stages, $\mu(K)$ is an element of the subdifferential $\partial_{x} Q(x)$ of the operating cost with respect to $x$ (see Appendix 2.1). This is a point to set mapping from $R_{+}^{|K|}$ into $R_{+}^{|K|}$ (Appendix 2.2). We can then restate $\mu(k, \ell)$ as $\mu(x ; k, \ell)$ (and $\mu(k)$ as $\mu(x ; k))$ in the above investment relation in order to express the dependence of the marginal value of capacity on the amount of capacity. The investment criterion is then written as

$$
0 \leq I(k)-\sum_{\ell \in L} \tau(\ell) \mu(x, k, \ell) \perp x(k) \geq 0
$$


or in abridged form after writing $\mu(x, k)=\sum_{\ell \in L} \tau(\ell), \mu(x, k, \ell)$ and collecting these expressions (11) for all $k$

$$
0 \leq I(K)-\mu(x, K) \perp x(K) \geq 0 .
$$

This complementarity relation summarizes the whole capacity investment model: assuming that one knows the mapping $\mu(x ; K)$ for a particular problem, the capacity expansion problem is entirely summarized in (12). The properties of the mapping $\mu(x, K)$ intervening in this model have important implications on the existence and unicity of equilibria. Because of the expository nature of this work, we only briefly mention these properties in passing and leave their detailed analysis for a further paper. We now extend this model to the stochastic case.

\subsection{Introducing risk}

The introduction of risk factors generalizes the above model to a stochastic environment. Fuel costs $c(k)$ and demand levels $d(\ell)$ are standard risk factors that immediately come to mind. Regulatory risks are new risks that take a prominent role in the current environment of the firm. They originate both from the regulation of the power sector and from related domains like environmental regulation. The cost $P C$, when interpreted as a price cap set by the Regulator is an illustration of regulatory risk: the Regulator sets the price during curtailment. We make no assumption on the dependence or independence of these risk factors. Let $\omega$ denote a scenario and $\Omega$ be the set of these scenarios. We note $c(k, \omega), d(\ell, \omega), P C(\omega)$ as the exogenous fuel cost, demand and $P C$ scenario. We also note $y(k, \ell, \omega), \mu(k, \omega), \pi(\ell, \omega)$ as the endogenous realisation of the primal and dual variables of the operation model in that scenario. The formulation of the optimization and complementarity problems extends to this more general problem by assuming risk neutral generators (investors) that see the same scenarios and share the same beliefs (probability $p(\omega)$ ) about their occurrence. This is stated as follows.

\subsubsection{The stochastic capacity expansion optimization model}

The second stage operations model in scenario $\omega$ becomes,

$$
Q(x ; \omega) \equiv \min _{y, z} \sum_{\ell \in L} \tau(\ell)\left[\sum_{k \in K} c(k ; \omega) y(k ; \ell, \omega)+P C(\omega) z(\ell, \omega)\right]
$$

s.t.

$$
0 \leq x(k)-y(k ; \ell, \omega) \quad \tau(\ell) \mu(k ; \ell, \omega)
$$




$$
\begin{gathered}
0 \leq \sum_{k \in K} y(k ; \ell, \omega)+z(\ell, \omega)-d(\ell, \omega) \quad \tau(\ell) \pi(\ell, \omega) \\
0 \leq y(k ; \ell, \omega) .
\end{gathered}
$$

The first stage investment part of the model is stated as

$$
\min _{x \geq 0} \sum_{k \in K} I(k) x(k)+E_{p} Q(x ; \omega) .
$$

where $E_{p}$ denotes the expectation under the $p$ measure. For notational convenience, we sometimes also refer to $E_{p} Q(x, \omega)$ as $Q(x)$.

\subsubsection{The stochastic capacity expansion equilibrium model}

As before, the equilibrium model is obtained by writing the KKT conditions of the second and first stage problems respectively. For the second stage problem, we have

$$
\begin{gathered}
0 \leq x(k)-y(k ; \ell, \omega) \perp \mu(k ; \ell, \omega) \geq 0 \\
0 \leq \sum_{k \in K} y(k ; \ell, \omega)+z(\ell, \omega)-d(\ell, \omega) \perp \pi(\ell, \omega) \geq 0 \\
0 \leq c(k ; \omega)+\mu(k ; \ell, \omega)-\pi(\ell, \omega) \perp y(k ; \ell, \omega) \geq 0 \\
0 \leq P C(\omega)-\pi(\ell, \omega) \perp z(\ell, \omega) \geq 0
\end{gathered}
$$

The first stage KKT conditions are stated as:

$$
0 \leq I(k)-E_{p} \sum_{\ell \in L} \tau(\ell) \mu(k ; \ell, \omega) \perp x(k) \geq 0
$$

or after explicitly introducing the dependence of $\mu(k ; \ell, \omega)$ on $x$, and rewriting $\mu(x, k, \omega)=$ $\sum_{\ell \in L} \tau(\ell) \mu(x ; k, \ell, \omega)$ and collecting all $k$ investment criteria

$$
0 \leq I(K)-E_{p} \mu(x, K, \omega) \perp x(K) \geq 0 .
$$

Again we sometimes also refer to $\mu(x, k)=E_{p} \mu(x, k, \omega)$ for the sake of notational convenience. Recall that $\mu(x ; K)$ is the $|K|$ dimensional mapping formed by the $\mu(x ; k)$. We refer to models of this type (relations (18) to (22) or relation (23)) as the Fixed Demand Model (FDM) .

\subsection{Focusing on the investment model}

The complementarity condition (23) summarizes the whole equilibrium description of capacity expansion in the stochastic case. It takes the form of a stochastic complementarity problem and could easily 
be extended to a stochastic variational inequality if there were constraints on the $x$ variables. While formulation (23) does not tell us anything that we could not have learned from the stochastic optimization model, it allows one to state questions that remain relevant when formulating an equilibrium model without being able to invoke an optimization problem to start with. The fundamental convexity property that underlies a significant part of the theory of stochastic programming carries through here by noting that $-\mu(x ; K, \ell)$ and hence $-\mu(x ; K)$ is monotone in $x$ (see Appendix 3 for the definition of a monotone operator). The complementarity condition (23) resembles the stochastic equilibrium model introduced in Gürkan et al. (1999). Note however that the mapping $-\mu(x ; K)$ is upper semi-continous and not continuous as in Gürkan et al. and in the more usual complementarity theory (see Appendix 2.2). The next section discusses variations of this model.

\section{Alternative equilibrium models of capacity expansion}

\subsection{Welfare maximization equilibrium models}

We noted that $-\mu(x ; K)$ is monotone in $x$ but not continuous. This creates technical difficulties if one wants to stick to the complementarity or variational inequality formulations, which are the more natural way to state equilibrium problems. The following introduces a variant of the model that leads to a continuous mapping $-\mu(x ; K)$ while at the same time being more in line with standard economic thinking. The common assumption in economic theory is to suppose that demand varies with prices. Even then, the common wisdom in electricity modelling is to admit that the demand of electricity is price insensitive in the short run, as is effectively the case in the short run operations model (18) to(21). This assumption is then commonly but unduly extended to the long run and embedded in the investment model. There are at least two reasons to question that extension. One is that demand, even if non price sensitive today in the short run, is clearly price sensitive in the long run, which is the natural horizon for a capacity expansion model. Large industrial consumers will not keep their demand unchanged in the long run if they anticipate high electricity prices. Similarly high electricity prices will induce conservation in household and tertiary and hence reduction of demand. The second reason is that the increasing interest for demand side management and the development of new technologies of the smart grid type will progressively introduce a true price response in the short run. Taking stock of these two reasons we modify the above model to encompass a demand function. We start with the optimization problem that we immediately state in a stochastic formulation. 
Let $d(p(\ell), \ell, \omega)$ be the demand function in time segment $\ell$, and scenario $\omega$. Both the demand and the price in time segment $\ell$ and scenario $\omega$ are one dimensional. Assuming, as in standard economics, that $d(\ell, \omega)$ is monotonically decreasing with $p(\ell, \omega)$, the function $d(p(\ell), \ell, \omega)$ can be inverted into $p(d(\ell), \ell, \omega)$ for each time segment $\ell$ and scenario $\omega$. We do not know much today about this demand function with several studies giving widely diverging results (e.g., Newbery, 2003 quotes a long run price elasticity of -1). It thus makes sense to embed the uncertainty on the demand function in the model, which justifies our introducing a dependence on $\omega$ in $d(p(\ell), \ell, \omega)$ and $p(d(\ell), \ell, \omega)$.

The stochastic optimization model of the short term welfare can then be stated as follows (in minimisation form) where MSTW stands for Minus Short Term Welfare:

$$
\operatorname{MSTW}(x, \omega) \equiv \min _{y} \sum_{\ell \in L} \tau(\ell)\left[\sum_{k \in K} c(k, \omega) y(k, \ell, \omega)-\int_{0}^{d(\ell, \omega)} p(\xi, \ell, \omega) d \xi\right]
$$

s.t.

$$
\begin{array}{cc}
0 \leq x(k)-y(k, \ell, \omega) & \tau(\ell) \mu(k, \ell) \\
0 \leq \sum_{k \in K} y(k, \ell, \omega)-d(\ell, \omega) & \tau(\ell) \pi(\ell) \\
0 \leq y(k, \ell, \omega) . &
\end{array}
$$

The long term welfare optimisation is similarly stated (in minimisation form) as

$$
\min _{x \geq 0} \sum_{k \in K} I(k) x(k)+E_{p} M S T W(x, \omega) .
$$

The equilibrium model is derived by writing the KKT conditions of that problem. We obtain for the short run market

$$
\begin{aligned}
& 0 \leq x(k)-y(k, \ell, \omega) \perp \mu(k, \ell, \omega) \geq 0 \\
& 0 \leq \sum_{k \in K} y(k, \ell, \omega)-d(\ell, \omega) \perp \pi(\ell, \omega) \geq 0 \\
& 0 \leq c(k, \omega)+\mu(k, \ell, \omega)-\pi(\ell, \omega) \perp y(k, \ell, \omega) \geq 0 \\
& 0 \leq \pi(\ell, \omega)-p(d(\ell), \ell, \omega) \perp d(\ell, \omega) \geq 0
\end{aligned}
$$

while the investment criterion becomes

$$
0 \leq I(k)-E_{p}\left[\sum_{\ell \in L} \tau(\ell) \mu(k, \ell, \omega)\right] \perp x(k) \geq 0 .
$$


or after introducing the dependence of $\mu(k, \ell, \omega)$ on $x$ and defining $\mu(x, k, \omega)=\sum_{\ell} \tau(\ell) \mu(x, k, \ell, \omega)$ and assembling these relations for all $k$

$$
0 \leq I(K)-E_{p} \mu(x, K, \omega) \perp x(K) \geq 0 .
$$

The main difference between (34) and (23) is in the properties of the mapping $\mu$ which is here a monotone continuous point to point mapping of the capacity $x$ (see Appendix 2.3). We refer to this model (relations (29) to (32) and (34)) as the Variable Demand Model (VDM).

\subsection{Optimization problems that do not extend to equilibrium models}

The above short run optimisation problems are of the convex type and hence have a well behave dual. This allows one to write KKT conditions that can easily be interpreted in terms of perfect competition equilibrium. Not all capacity expansion optimization models have a convex second stage optimization problem. This is in particular the case when the second stage involves unit commitment features (start up and shutdown of machines, minimum down and up time constraints). Considerable effort has been devoted to the analysis of these questions in short term and hedging models (e.g. Eichhorn et al., 2010, Kuhn and Schultz, 2009, and Römisch and Vigerske, 2010). We are not aware of any attempt to include them in capacity expansion models. Second stage optimization models that include unit commitment features cannot be converted into complementarity problems and hence in complementarity models of equilibrium. Convexification of these effects such as elaborated in Gribik et al. (2007) could however lead to approximate equilibrium models.

\subsection{Equilibrium models that do not derive from optimization problems}

Leaving aside second stage optimisation problems that cannot be exactly converted into equilibrium models, we now consider equilibrium problems that cannot be obtained from KKT conditions of optimisation problems. These abound in the electricity restructuring because of the diversity of organisations of the electricity market and its numerous submarkets (e.g., transmission, reserve of different quality). Different arrangements of other related markets such as the EU-ETS also easily lead to short run equilibrium models that do not derive from optimization (e.g. Ehrenmann and Smeers, 2010). In the interest of space we do not engage into that discussion here but simply illustrate our point by presenting an extension of the welfare maximisation problem described by equations (29) to (33) to a model where the demand model cannot be integrated into a willingness to pay function. This problem originates in the 
PIES model (Ahn and Hogan, 1982) that was built by combining a large linear programming model and various heterogenous demand models that could not be integrated into a utility function (see Harker and Pang, 1990, for the integrability property).

This variant of the equilibrium model can be stated as follows. Let $D(\omega)=(d(\ell, \omega), \ell \in L)$ and $P(\omega)=(p(\ell, \omega), \ell \in L)$ denote the vectors of demand and price in the different time segments. Smart grid technologies aim, among other goals, at introducing storage possibilities across the different time segments. In other words, demand $d(\ell, \omega)$ in time segment $\ell$, no longer depends on the sole price $p(\ell, \omega)$ in time segment $\ell$, but on the whole price vector $P(\omega)$. The objective is to create at the demand side, storage possibilities that are so difficult to achieve at the generation side. Taking stock of that extension, we write the demand model as

$$
D(P, \omega) \equiv\{d(P, \ell, \omega), \ell \in L\} .
$$

Assuming that the vector function $D(P, \omega)$ can be inverted into a system $P(D, \omega)$

$$
P(D, \omega) \equiv\{p(D, \ell, \omega), \ell \in L\}
$$

but that $P(D, \omega)$ cannot be integrated into a willingness to pay function (equivalently $P(D, \omega)$ is not a gradient function) we replace the short run welfare maximization problem by the following equilibrium conditions.

$$
\begin{aligned}
& 0 \leq x(k)-y(k, \ell, \omega) \perp \mu(k, \ell, \omega) \geq 0 \\
& 0 \leq \sum_{k \in K} y(k, \ell, \omega)-d(\ell, \omega) \perp \pi(\ell, \omega) \geq 0 \\
& 0 \leq c(k, \omega)+\mu(k, \ell, \omega)-\pi(\ell, \omega) \perp y(k, \ell, \omega) \geq 0 \\
& 0 \leq \pi(\ell, \omega)-p(D, \ell, \omega) \perp d(\ell, \omega) \geq 0
\end{aligned}
$$

These conditions cannot be obtained as KKT conditions of a welfare maximization problem. Still the investment criterion remains

$$
0 \leq I(K)-E_{p}\left[\sum_{\ell \in L} \tau(\ell) \mu(K, \ell, \omega)\right] \perp x(K) \geq 0 .
$$

Applying a well known integrability theorem (e.g. Theorem 1.3.1 in Facchinei and Pang, 2003), one can show that the model would have been a stochastic optimization problem if the inverted demand system $P(D, \omega)$ were integrable, that is, if it satisfied

$$
\frac{\partial p(D, \ell, \omega)}{\partial d\left(\ell^{\prime}, \omega\right)}=\frac{\partial p\left(D, \ell^{\prime}, \omega\right)}{\partial d(\ell, \omega)} \text { for all } \ell, \ell^{\prime},
$$


This condition was not satisfied in the PIES model (Ahn and Hogan, 1982); it will generally not be satisfied in multiperiod investments models where the demand models is "adapted" (demand in some period depends on past prices but not on future prices (e.g. Wu and Fuller, 1996) and hence does not derive from a multiperiod willingness to pay function. Even in single period investment model the demand shifting properties created by smart grids will violate this integrability property. But even though we cannot rely on an optimization model, the investment problem retains the standard complementarity form (39) or with the usual short cut

$$
0 \leq I(K)-\mu(x ; K) \perp x(K) \geq 0 .
$$

Note that any demand system $P(D, \omega)$ that can be written as a gradient function satisfies the integrability property. This is in particular the case of multiperiod perfect foresight demand systems. Problem (40) can then be reformulated as an optimization model. The mapping $\mu(x ; K)$ is continuous in $x$. Its monotonicity properties depend on the demand system. We do not discuss the question here (see Aghassi et al., 2006 for a discussion of convexity of asymmetric variational inequality problems).

\section{Project finance and asset specific discounting rates}

Investments in a risky environment require risk adjusted discount factors. Specifically the two-stage investment model (1) to (5) of section 1.1 requires converting the total plant investment cost $\mathcal{I}(k)$ into the annual investment cost $I(k)$. This is commonly done, using the standard formula

$$
I(k) \sum_{t=1}^{T} \frac{1}{(1+r(k))^{t}}=\mathcal{I}(k) \quad(\text { in } € / \mathrm{Mw})
$$

where $T$ is the assumed life of the plant and $r(k)$ is a discount rate. This annual investment cost can be turned into an hourly capacity cost in $€ /$ Mwh by dividing by 8760 (the number of hours in a year).

The tradition in the regulated industry is to apply a single discount rate $r$ to all equipment in capacity expansion models of the type described by relations ((1) to (5)). This discount rate reflects the risk exposure of the company and is meant to provide the adequate remuneration of investors and lenders. In order to simplify the discussion we assume a full equity financing (no debt) which means that $r$ represents the cost of equity. Cost plus regulation limited the risk bearing on the monopoly company and the Regulator accordingly decided the discount rate and the allowed return on capital of the company. The long life of the plants, the slowly evolving nature of the portfolio and the relatively surprise free evolution of the 
economy in a still recent past further contributed to reduce risk.

The restructuring of the sector and the introduction of a project finance approach in investment changed this practice. A generation unit is valued on the basis of its own merit, which in particular means on the basis of its own risk exposure. The plant thus has its own risk adjusted discount factor. A common practice (Graham and Harvey, 2001) is to derive these risk factors through a CAPM based approach, applied this time to each plant separately. The CAPM theory of financial assets is extensively discussed in any textbook of corporate finance; in contrast its application to physical assets is much less elaborated. A comprehensive treatment and appropriate references can be found in Armitage (2005), which points to several drawbacks of the use of the CAPM for project evaluation. Notwithstanding the reservations against the CAPM and its standard applications to physical assets found in the literature, we conduct the discussion in these terms because of the wide application of the method in practice.

The insertion of the project finance view in capacity expansion models raises different questions. Some are due to the multiperiod discounting of the cash flows. For the sake of brevity and in order to stick to our two stage set up, we do not discuss them here and refer the reader to a companion paper (Ehrenmann and Smeers (2009)). Other questions already arise in the two stage context adopted in this paper. Suppose that $I(k)$ is determined from the total investment $\operatorname{cost} \mathcal{I}(k)$ of equipment $k$ using formula (41). Both the Fixed Demand (FDM: relation: (23)) or Variable Demand models (VDM: relation (34)) can be formally implemented by assuming exogenously determined $r(k)$. This does not pose particular computational difficulty in a two-stage model. The question discussed below is whether it is a consistent treatment of risk.

We first note that the short term equilibrium models (relations (18) to (21) for FDM and relations (29) to (32) for VDM) are unaffected by the choice of the discount rate. Each machine only appears in these short term models through its capacity and variable cost; the market therefore finds the short term equilibrium irrespectively of the investment cost of these units. Things are different in the investment model ((23) for FDM and (32) for VDM). The analogy (see Armitage (2005), chapter 6, for a treatment that goes beyond the analogy) between financial and physical assets that underlies the application of the CAPM to physical assets suggests the following reasoning. A unitary investment in plant $k$ buys a 
capacity $\frac{1}{I(k)}$, a unit capacity in that plant generates a payoff

$$
\sum_{\ell} \tau(\ell)[\pi(\ell, \omega)-c(k, \ell, \omega)] y(k, \ell, \omega)
$$

Combining the two relations, the net return on investment in scenario $\omega$ of a unit investment in plant $k$ can be written as

$$
R(k, \omega)=\frac{\sum_{\ell} \tau(\ell)[\pi(\ell, \omega)-c(k, \ell, \omega)] y(k, \ell, \omega)}{I(k)} .
$$

Note that $R(k, \omega)$ and hence $E_{p}[R(k, \omega)]$ are results of the model established with given $r(k)$. The question is whether the returns $R(k, \omega)$ are compatible with the discount rate $r(k)$ used for the equipment or in other words whether $r(k)=E_{p}[R(k, \omega)]-1$. This requires explicitly invoking the underlying risk theory, which in this section is the usual CAPM.

In order to do so we expand the definition of the scenarios by explicitly introducing the "market" $M(\omega)$ and the return on the market $R(M, \omega)$ in scenario $\omega$. These are additional data that need to be part of the scenarios. Limiting the discussion to the fixed demand model for the sake of brevity, scenario $\omega$ therefore encompasses assumptions on $c(k, \omega), d(\ell, \omega), P C(\omega), M(\omega)$ and $R(M, \omega)$. In order to be consistent with the CAPM, $r(k)$ should be consistent with the return $R(k, \omega)$ accruing from an investment in plant $k$. This implies imposing the standard CAPM formula

$$
r(k)=r^{f}+\frac{\operatorname{cov}(R(k, \omega), R(M, \omega))}{\sigma^{2}[R(M, \omega)]} E_{p}\left[R(M, \omega)-R^{f}\right]
$$

where $R^{f}$ is the gross risk free rate ( $1+$ the net risk free rate $\left.r^{f}\right)$. The investment models (23)(for FDM) or (34)(for VDM) in a CAPM based environment are then completed by adding the two relations

$$
I(k) \sum_{t=1}^{T} \frac{1}{(1+r(k))^{t}}=\mathcal{I}(k) \quad(\text { in } € / \mathrm{Mw})
$$

where $r(k)$ is satisfies

$$
r(k)=r^{f}+\frac{\operatorname{cov}(R(k, \omega) R(M, \omega))}{\sigma^{2}[R(M, \omega)]} E_{p}\left[R(M, \omega)-R^{f}\right] .
$$

One can immediately see that the addition of these relations destroys the two stage decomposition of the model by introducing a strong coupling between these stages. It also destroys any monotonicity property. The model can however still be posed as a fixed point problem of $r(K)$. 


\section{Linear stochastic discount factors}

The above approach requires solving a fixed point problem (a "circularity" problem in Armitage, 2005) in order to find the $r(k)$ ) compatible with the endogenous risk exposure of the different plants. This is cumbersome. An alternative much lighter method introduced in Fama (1997) is to resort to CAPM based deterministic equivalents of stochastic cash flows. We present this approach in the context of the more general theory of linear stochastic discount factors that embeds not only the CAPM but also the less used Arbitrage Pricing Theory (APT) and the multitemporal consumer theory. As before we assume an expanded definition of scenarios that includes the "market" $M(\omega)$. We refer the reader to Cochrane (2005) or Armitage (2005) for discussions of linear stochastic discount rates and restrict ourselves to the elements of the theory necessary for constructing the stochastic equilibrium models.

\subsection{A primer on linear stochastic discount factors}

Consider a two stage set up where one wants to assess in time 0 a cash flow $X(\omega)$ accruing in time 1 . A stochastic discount factor(or price kernel or state price vector) is a vector $m(\omega)$ such that the value of this cash flow in stage 0 is equal to $E_{p}[m(\omega) \times X(\omega)]$. Both economic and finance offer theories that allow one to construct the price kernel $m(\omega)$. Specifically (e.g. see Cochrane, 2005) the CAPM leads to state a stochastic discount rate of the form

$$
m(\omega)=a-b \times R(M, \omega)
$$

where $a$ and $b$ are determined to satisfy

$$
E_{p}[m(\omega) \mid \omega]=\frac{1}{R_{f}}
$$

$(m(\omega)$ prices the risk free asset $1(\omega)$ that redeems 1 in all scenarios $\omega)$, and

$$
E_{p}[m(\omega) \times R(M, \omega)]=\frac{1}{R_{f}}
$$

( $m(\omega)$ prices the "market" $M(\omega))$. Because $R(M, \omega)$ and $R_{f}$ are data of the problem, $a$ and $b$ and hence the stochastic discount factor are also data to the problem.

The pricing kernel $m(\omega)$ and the payoff $X(\omega)$ do not necessarily span the same space. Let $X=$ $X_{m}+X_{\perp m}$ be a decomposition of the space of payoffs where $X_{m}$ is the subspace spanned by $m(\omega)$ (which has a nonzero covariance with $m(\omega)$ ) and $X_{\perp m}$ the subspace orthogonal to $X_{m}$ with respect to 
the scalar product $E_{p}(x \times y)$. In standard financial language, $X_{m}(\omega)$ is the systematic risk embedded in the payoff $X(\omega)$ (the risk priced by $m(\omega)$ or the "market" in CAPM parlance) and $X_{\perp m}(\omega)$ is the idiosyncratic component of $X(\omega)$, that is the component that is priced to zero by $m(\omega)$.

We first explain that resorting to linear discount rates allows one to conduct all discounting at the risk free rate and therefore eliminates the need for finding a risk adjusted discount rate that reflects the risk exposure of the plants (in particular in order to find the annual value $I(k)$ ). We thus bypass the need to solve a fixed point problem. In order to conduct the discussion with sufficient generality, we depart from the two stage paradigm and consider a three stage set up where one invests in period 0 and collects random payoffs $X^{1}\left(\omega_{1}\right)$ and $X^{2}\left(\omega_{1}, \omega_{2}\right)$ in periods 1 and 2. The stochastic discounting approach extends as follows. Let $m^{1}\left(\omega_{1}\right)$ and $m^{2}\left(\omega_{2} \mid \omega_{1}\right)$ be the stochastic and conditional stochastic discount factors in stages 1 and 2 respectively. Let also $p^{1}\left(\omega_{1}\right)$ and $p^{2}\left(\omega_{2} \mid \omega_{1}\right)$ be the probabilities and conditional probabilities of the different states of the world in stages 1 and 2 respectively. Proceeding recursively from stage 2 to 0 , the global value in 0 of the two cash flows is equal to

$$
E_{p^{1}}\left[m^{1}\left(\omega_{1}\right) \times\left[X^{1}\left(\omega_{1}\right)+E_{p^{2}\left(\mid \omega_{1}\right)}\left[m^{2}\left(\omega_{2} \mid \omega_{1}\right) \times X^{2}\left(\omega_{2} \mid \omega_{1}\right)\right)\right]\right] .
$$

We now show how this expression can be restated in a form that only involves the risk free discount factor.

The value in stage 1 of payoff $X^{2}\left(\omega_{2} \mid \omega_{1}\right)$ conditional on $\omega_{1}$ can be written

$$
\begin{aligned}
& E_{p^{2}\left(\mid \omega_{1}\right)}\left[m_{2}\left(\omega_{2} \mid \omega_{1}\right) X^{2}\left(\omega_{2} \mid \omega_{1}\right)\right] \\
= & \frac{1}{R^{f}}\left[E_{p^{2}\left(\mid \omega_{1}\right)} X^{2}\left(\omega_{2} \mid \omega_{1}\right)+R^{f} \operatorname{cov}\left[m_{2}\left(\omega_{2} \mid \omega_{1}\right), X^{2}\left(\omega_{2} \mid \omega_{1}\right)\right]\right] \\
= & \frac{1}{R^{f}} \widetilde{X}^{2}\left(\omega_{1}\right)
\end{aligned}
$$

where $\widetilde{X}^{2}\left(\omega_{1}\right)$ is a deterministic equivalent in period 1 of the conditional cash flow $X^{2}\left(\omega_{2} \mid \omega_{1}\right)$. The payoff accruing in stage 1 and to be assessed in stage 0 is thus

$$
X^{1}\left(\omega_{1}\right)+\frac{1}{R^{f}} \tilde{X}^{2}\left(\omega_{1}\right)
$$

Conducting the same reasoning, the valuation in stage 0 of this random cash flow in stage 1 can be written as

$$
\frac{1}{R^{f}} \tilde{X}^{1,2}
$$

One can now restate these manipulations by expliciting the different contributions accruing in stages 1 
and 2. Specifically

$$
\widetilde{X}^{2}\left(\omega_{1}\right)=E_{p^{2}\left(\mid \omega_{1}\right)} X^{2}\left(\omega_{2} \mid \omega_{1}\right)+R^{f} \operatorname{cov}\left[m_{2}\left(\omega_{2} \mid \omega_{1}\right), X^{2}\left(\omega_{2} \mid \omega_{1}\right)\right]
$$

is the deterministic equivalent in stage 2 of the conditional random cash flow $X_{2}\left(\omega_{2} \mid \omega_{1}\right)$. Similarly

$$
\widetilde{X}^{1}\left(\omega_{1}\right)=E_{p^{1}} X^{1}\left(\omega_{1}\right)+R^{f} \operatorname{cov}\left[m_{1}\left(\omega_{1}\right), X^{1}\left(\omega_{1}\right)\right]
$$

is the deterministic equivalent in stage 1 (before discounting to 0 by $\frac{1}{R^{f}}$ ) of the random cash flow $X_{1}(\omega)$. One observes that all discounting can be conducted at the risk free rate using CAPM based deterministic equivalent cash flow (see Armitage, 2005 for a detailed treatment). This justifies conducting all the valuations at the risk free rate by working with risk adjusted cash flows. The approach bypasses the "circularity" of the standard discounting practice, here presented through a fixed point model. This is the approach taken in the next subsection. It avoids resorting to the annoying fixed point problem and is always compatible with the CAPM theory. In contrast the more standard discounting approach suffers from other circularity aspects arising when projects are also partially financed by debt (Fama, 1997). Last we shall see that the certainty equivalent cash flow offers a good chance (but no guarantee) of retaining the monotonicity properties of $\mu(x ; k)$.

The models of Sections 4.1 and 3 are equivalent in this two-stage context, provided one makes the assumption that the annual investment cost can be obtained by a single $r(k)$ (unconditional CAPM). This equivalence no longer holds in more general multiperiod cases where the certainty equivalent approach (Fama, 1977) can always be applied but the risk adjusted discount rate requires some assumptions. We thus follow the certainly-equivalent approach (Fama, 1977) that even though less usual, does not suffer from the criticism addressed to the standard risk adjusted discounting.

\subsection{Optimization and equilibrium models}

Returning to the reference two stage set up, we assume that the annuities $I(k)$ of plants $k$ have been computed from the total investment $\operatorname{costs} \mathcal{I}(k)$ at the risk free rate $R^{f}$. We again begin with the optimization form of the capacity expansion problem where the second stage objective function $Q(x, \omega)$ is given by the short run model (2) to (4). Calling upon the notion of stochastic discount factor the value in stage 0 of the total random cost incurred in stage 1 can be stated as $R^{f} E_{p}[m(\omega) Q(x, \omega)]$ where $m(\omega)$ 
is the stochastic discount factor. The minimization of the total investment and operations cost can then be stated as

$$
\min _{x \geq 0} \sum_{k \in K} I(k) x(k)+R^{f} E_{p}[m(\omega) Q(x, \omega)]
$$

or

$$
\min _{x \geq 0} \sum_{k \in K} I(k) x(k)+E_{p}\left[Q(x, \omega)+R^{f} \operatorname{cov}[m(\omega), Q(x, \omega)] .\right.
$$

Recall that the state price $m(\omega)$ is exogenous to the problem(as in a CAPM based formulation) and that the expectation with the price kernel is a linear operator that therefore introduces minimal complications. The consequence is that the problem stated in the original variables $x, y$ and $z$ retains the standard form of a convex stochastic optimization problem for which one can write KKT conditions. Because the definition of $Q(x, \omega)$ remains unchanged, its KKT conditions are also unchanged: they are restated below for the Fixed Demand Model

$$
\begin{gathered}
0 \leq x(k)-y(k ; \ell, \omega) \perp \mu(k ; \ell, \omega) \geq 0 \\
0 \leq \sum_{k \in K} y(k ; \ell, \omega)+z(\ell, \omega)-d(\ell, \omega) \perp \pi(\ell, \omega) \geq 0 \\
0 \leq c(k ; \omega)+\mu(k ; \ell, \omega)-\pi(\ell, \omega) \perp y(k ; \ell, \omega) \geq 0 \\
0 \leq P C(\omega)-\pi(\ell, \omega) \perp z(\ell, \omega) \geq 0
\end{gathered}
$$

The model is completed by the KKT conditions of the investment optimization problem. These become (using the abridged notation $\mu(x, k, \omega)$ )

$$
0 \leq I(K)-R^{f} E_{p}[m(\omega) \mu(x, K, \omega)] \perp x(K) \geq 0 .
$$

Because $-\mu(x, K, \omega)$ is a monotone operator, the expression $-E_{p}[m(\omega) \mu(x, K, \ell, \omega)]$ would then also be a monotone operator (and problem (47) a convex problem in $x$ ) if the stochastic discount rate $m(\omega)$ were non negative. This is not guaranteed, for instance for the stochastic discount rate derived from the CAPM. It can then happen that the optimization problem (47) is unbounded because of those scenarios $\omega$ where $m(\omega)$ is negative. From an optimization point of view an unbounded problem does not have any dual solution. From an equilibrium point of view, $-E_{p}[m(\omega) \mu(x, K, \ell, \omega)]$ is no longer monotone and (in this particular case) (53) has no solution. The origin of the problem is that the commonly used CAPM method does not necessarily guarantee that all $m(\omega)$ are non negative (it does not satisfy the stochastic dominance property). This is a matter of data on $R(M, \omega)$. The occurrence of negative $m(\omega)$ is thus entirely a CAPM matter and is independent of the power problem on hand; this can be checked ex ante. 
Finally note that even though all annual investment costs $I(k)$ are computed from the total investment cost $\mathcal{I}(k)$ (including intermediary financial costs during construction) at a common risk free rate, and the same state price $m(\omega)$ applies to all plants, the formulation effectively accounts for the risk exposure specific to each plant. The method is thus fully in line with a CAPM based project finance approach that values each plant according to its risk exposure. This consistence is achieved through the deterministic equivalent cash flow.

$$
E_{p}[m(\omega) \mu(x, k, \omega)]=E_{p}[\mu(x, k, \omega)]+R^{f} \operatorname{cov}[m(\omega), \mu(x, k, \omega)]
$$

where $\operatorname{cov}[m(\omega), \mu(x, k, \omega)]$ is the plant specific risk adjustment to the expected gross margin.

\section{Non-linear stochastic discount rates and valuation functions}

Linear stochastic discount factors price systematic risk but not idiosyncratic risk. The capacity expansion model discussed in the preceding section is based on linear stochastic discount factors and hence only prices systematic risk. The equilibrium model therefore complies with economic theories that claim that idiosyncratic risk can only have zero price at equilibrium and hence can be discarded. It does not fit with other economic theories that explain that agency costs allow idiosyncratic risk to intervene in decisions. We assume in this section that agency costs may lead investors to account for idiosyncratic risk in their decisions. Linear discount factors that only span a subspace of the payoffs cannot, by construction, reflect that phenomenon. The modern theory of risk functions allows one to expand the above equilibrium model by introducing non-linear stochastic discount factors that we here apply to the subspace of the payoffs that are not priced by $m(\omega)$. The theory of coherent risk functions originates in Artzner (1999); an extensive treatment of these functions in optimization is provided in Shapiro et al. (2009). We here insert coherent risk functions in equilibrium models. Because of the context of our model (focussing more on positive cash flows than positive losses) we conduct the discussion in terms of both risk and valuation functions; depending on the context we want to find the "value" of a cash flow or the "risk" of a cost.

\subsection{A primer on coherent risk/valuation functions}

Consider a two periods problem where one wants to assess in stage 0 a cash flow $X(\omega)$ accruing in stage 1. A valuation function $\rho(X(\omega))$ gives a value to this cash flow. In order to remain as close as possible to the above discussion of stochastic discount factors we limit ourselves to coherent valuation 
functions introduced by Artzner et al. (1999) in a context of risk and now extensively developped in the literature. Coherent risk/valuation functions $\rho$ satisfy four axioms that are recalled in Appendix 4 . We are particularly interested in a representation theorem that states that every coherent valuation function can be represented as

$$
\rho(X(\omega))=\operatorname{Inf}_{m \in \mathcal{M}} E_{p}[m(\omega) \times X(\omega)] .
$$

where $\mathcal{M}$ is a convex set of probability measures.

The representation theorem implies that a valuation function generates a stochastic discount factor $m(\omega)$ which is the minimant of the above infimum problem. This stochastic discount factor differs from the one discussed in the preceding section in at least two respects. Because $m$ is a probability measure, it is non negative. This guarantees the monotonicity property (Axiom 2 in Appendix 4) that is sometimes violated in stochastic discount factors derived from other theories such as the CAPM. Another major difference is that this stochastic discount rate is a function of the payoff $X(\omega)$ and hence the valuation process is a non-linear operator.

We accordingly write $\rho(X(\omega))=E_{p}[m(X, \omega) \times X(\omega)]$ and note that $\rho(X(\omega))$ is a concave function of $X$ in the case of the risk adjusted valuation of a payoff (and a convex function for the risk adjusted valuation of a cost). We also note that one of the axioms defining risk/valuation functions (Axiom 3 in Appendix 4) immediately implies that the value of a risk free asset $1(\omega)$ that redeems 1 in all states of the world in stage 1 is equal to $\frac{1}{R^{f}}$, or in other words $\rho(1(\omega))=\frac{1}{R^{f}}$. The reader is referred to the original papers of Artzner et al. (1999) and to the extensive discussion and the many examples of risk functions (coherent or not) found in Shapiro et al. (2009). All this applies here whether to risk or value functions.

We conclude this brief summary by noting that, because risk/valuation functions give rise to stochastic discount factors, they also allow one to restate the value of a random cash flow as a deterministic equivalent where all discounting takes place at the risk free rate. We briefly recall the reasoning developed in the previous Section 4.1 and adapt it to this non-linear case. We caution however that the multitemporal discounting with risk/valuation functions raises questions of time consistency of these functions that are not discussed here (see Artzner et al. (2002) and Shapiro et al. (2009)). In order to justify the discounting at the risk free rate consider again a three stages set up where one invests in period 0 and collects the random payoffs $X^{1}(\omega)$ and $X^{2}\left(\omega_{1}, \omega_{2}\right)$ in stages 1 and 2 respectively. Let $m^{2}\left(X^{2}\left|\omega_{1}, \omega_{2}\right| \omega_{1}\right)$ 
be the conditional stochastic discount factor derived from the valuation function in stage 2 for given $\omega_{1}$. As before let $p^{2}\left(\omega_{2} \mid \omega_{1}\right)$ be the conditional probability of the different states of the world in stage 2 . The value in stage 1 of payoff $X^{2}\left(\omega_{2} \mid \omega_{1}\right)$ conditional on $\omega_{1}$ can be written

$$
\begin{aligned}
& E_{p^{2} \mid \omega_{1}}\left[m_{2}\left(X^{2}\left|\omega_{1}, \omega_{2}\right| \omega_{1}\right) \times X^{2}\left(\omega_{2} \mid \omega_{1}\right)\right] \\
= & \frac{1}{R^{f}}\left[E_{p^{2} \mid \omega_{1}} X^{2}\left(\omega_{2} \mid \omega_{1}\right)+R^{f} \operatorname{cov}\left[m_{2}\left(X^{2}\left|\omega_{1}, \omega_{2}\right| \omega_{1}\right), X^{2}\left(\omega_{2} \mid \omega_{1}\right)\right]\right] \\
= & \frac{1}{R^{f}} \widetilde{X}^{2}\left(\omega_{1}\right)
\end{aligned}
$$

where $\widetilde{X}^{2}\left(\omega_{1}\right)$ is the deterministic equivalent in period 1 of the conditional cash flow $X^{2}\left(\omega_{2} \mid \omega_{1}\right)$.

Let now $p^{1}\left(\omega_{1}\right)$ be the probability measure of the different states of the world in stage 1 . We need to assess in stage 0 a payoff

$$
X^{1}\left(\omega_{1}\right)+\frac{1}{R^{f}} \widetilde{X}^{2}\left(\omega_{1}\right)
$$

accruing in period 1 . Conducting the same reasoning, the valuation in stage 0 of this random cash flow accruing in stage 1 can be written as

$$
\frac{1}{R^{f}} \widetilde{X}^{1,2}
$$

As in Section 4.1, one sees that all deterministic equivalent cash flows are discounted at the risk free rate provided these deterministic equivalents have been computed with the stochastic discount factors derived from the valuation function. The implication is that the annual investment cost $I(k)$ of a plant $k$ can be computed from the total investment cost $\mathcal{I}(k)$ using the risk free rate. This part of the reasoning would remain true if we were to account for the question of time consistency alluded to above. But the multitemporal discounting of the cash-flows would be restricted to time consistent valuation functions.

\subsection{Coherent risk/valuation functions and stochastic discount factors}

Consider again the decomposition of the payoff space $X=X_{m}+X_{\perp m}$ where $X_{m}(\omega)$ and $X_{\perp m}(\omega)$ are respectively the systematic and idiosyncratic components of the payoff. Different economic theories (e.g. CAPM, APT, multitemporal consumption model) can be used to construct a stochastic discount rate $m^{\star}(\omega)$ that prices the systematic risk while giving a zero value to the idiosyncratic risk. We introduce a price for the idiosyncratic risk embedded in $X(\omega)$ by writing the value of the payoff in stage zero as

$$
\rho(X(\omega))=E_{p}\left[m^{\star}(\omega) \times X_{m^{\star}}(\omega)\right]+\operatorname{Inf}_{m \in \mathcal{M}_{\perp m^{\star}}} E_{p}\left[m(\omega) \times X_{\perp m^{\star}}(\omega)\right] .
$$


where $m^{\star}$ prices the systematic risk (for instance as specified in the CAPM) and $\mathcal{M}_{\perp m^{\star}}$ is a set of probability measures of $\mathcal{M}$ orthogonal to $m^{\star}$ that price the idiosyncratic risk.

This expression requires a decomposition of the payoff into its systematic (priced by $m^{\star}(\omega)$ ) and idiosyncratic parts (orthogonal to $m^{\star}(\omega)$ ). This is easily obtained by writing $X_{m}^{\star}(\omega)$ as the projection

of $X(\omega)$ on $m^{\star}$, or $X(\omega)=\frac{\operatorname{cov}\left[X(\omega) m^{*}(\omega)\right]}{\sigma^{2}\left(m^{*}(\omega)\right)} m^{*}(\omega)+X_{\perp m^{*}}(\omega)$. The obtained expression $X_{\perp m^{*}}(\omega)$ is clearly linear in $X(\omega)$.

\subsection{Optimization model}

Consider now the risk adjusted capacity expansion optimization model. This model is expressed in terms of costs, which justifies our using the risk interpretation of $\rho$. We thus apply the above risk function to the second stage cost $Q(x, \omega)$ and define the following convex optimization problem.

$$
\min _{x \geq 0} \sum_{k \in K} I(k) x(k)+R^{f} \rho[Q(x, \omega)]
$$

One obtains the KKT conditions of that optimization problem by concatenating the equilibrium conditions of the short-term problem (18) - (21) with the following risk adjusted investment condition

$$
0 \leq I(K)-R^{f} \frac{\partial \rho}{\partial \nu(\omega)} \frac{\partial Q(x, \omega)}{\partial x} \perp x(K) \geq 0
$$

Define the stochastic discount factor $\phi(x, \omega)=\frac{\partial \rho(Q(x, \omega))}{\partial \nu(\omega)}=m^{\star}(\omega)+m(Q(x, \omega))$ and recall that $\frac{\partial Q(x, \omega)}{\partial x(k)}=\mu(x, k, \omega)$. The investment criterion is stated as

$$
0 \leq I(K)-R^{f} E_{p}(\phi(x, \omega) \times \mu(x, K, \omega)) \perp x(K) \geq 0 .
$$

Note that the representation theorem implies that $m(Q(x, \omega))$ is a probability measure. In contrast $m^{\star}$ need not be as the example of the CAPM discussed in the preceding section shows. $\mu(x ; K)=$ $E_{p}(\phi(x, \omega) \times \mu(x, K, \omega))$ is a monotone operator when $m^{\star}$ is non negative since $\rho(X(\omega))$ is then a coherent risk function (see Appendix 4).

\subsection{Equilibrium models}

The above formulation cannot be directly restated in competitive equilibrium terms. Specifically the dependence of the stochastic discount rate on the total system cost or welfare has a natural interpretation 
for a risk averse monopoly company but it does not apply to agents of a market where the total cost of the system is the sum of the costs incurred by these agents. We first discuss tentative equilibrium interpretations of condition (58) by invoking different assumptions of market structures.

Suppose without loss of generality that the market only comprises a single electricity consumer (noted $c$ ) and the other agents (noted $o \in O)$ own generation capacities $x^{o}(k), k \in K\left(x(k)=\sum_{o \in O} x^{o}(k)\right.$, for all $k$ ). Take first the Fixed Demand Model; the short run cost optimization model (of section 1.3) in state of world $\omega$ can be restated in dual form as

$$
Q(x, \omega) \equiv \max \sum_{\ell} \tau(\ell)\left[\pi(\ell, \omega) d(\ell, \omega)-\sum_{k} \mu(k, \ell, \omega) x(k)\right]
$$

s.t.

$$
\begin{aligned}
& \pi(\ell, \omega)-\mu(k, \ell, \omega) \leq c(k, \omega) \\
& \pi(\ell, \omega) \leq P C(\omega) \\
& \pi(\ell, \omega) \geq 0, \mu(k, \ell, \omega) \geq 0 .
\end{aligned}
$$

The dual objective function is the sum of the total payment for electricity by the consumer $\Pi(\omega)=$ $\sum_{\ell} \tau(\ell) \pi(\ell, \omega) d(\ell, \omega)$ minus the sum over all plants of the gross margins $\mu(x, k, \omega) x^{o}(k)$ made by the owners of the different capacities $x^{o}(k)$. The total profit of capacity owner $o$ is then $\Pi^{o}(x, \omega)=$ $\sum_{k} \mu(x, k, \omega) x^{o}(k)$.

We write

$$
Q(x, \omega)=\Pi(\omega)-\sum_{o \in O} \Pi^{o}(x, \omega) .
$$

Recalling that the derivative of $Q(x, \omega)$ with respect to $x(k)$ is the marginal profit $\mu(x, k, \omega)$ accruing to every owner of capacity $k$ in state of the world $\omega$ when increasing this capacity, the investment condition (59) could be associated to an owner $o$ of capacity $k$ if we could ascertain that $\frac{\partial \rho[Q(x, \omega)]}{\partial \nu(\omega)}=\phi(x, \omega)$ is the stochastic discount rate of every capacity owner $o$ making a profit $\sum_{k} \mu(x, k, \omega) x^{o}(k)$. It remains to identify if and when this latter condition can hold.

Consider first the case where all capacity owners $o$ have the same generation structure $x^{o}$, that is the same portfolio of generation plants (generators are identical up to a scaling factor). Their profits $\Pi^{0}(x, \omega)$ are then equal for all $\omega$ up to the scaling factor. Suppose also that they have the same risk function. Let $\phi^{o}\left(\Pi^{o}(x, \omega), \omega\right)$ be the stochastic discount factor of capacity owner $o$ when it is making a 
profit $\Pi^{o}(x, \omega)=\sum_{k} \mu(x, k, \omega) x^{o}(k)$. Because these generators have the same share of different plants, the positive homogeneity of the coherent risk function $\rho$ (Axiom 4 in Appendix 4) implies they also have the same stochastic discount factor. Unfortunately, this common value is not necessarily equal to $\frac{\partial \rho[Q(x, \omega)]}{\partial \nu(\omega)}$ because the total cost $Q(x, \omega)$ which is the argument of the risk function in (58) contains the term $\Pi(\omega)$ which is not proportional to $\Pi^{0}(x, \omega)$.

Consider now consumer $c$. The stochastic discount rate of this consumer is $\phi^{c}(\Pi(\omega), \omega)$, which is equal to $\frac{\partial \rho(\Pi(\omega))}{\partial \nu(\omega)}$. Suppose now that we can arrange for

$$
\phi^{o}\left(\Pi^{o}(x, \omega), \omega\right)=\phi^{c}(\Pi(\omega), \omega) \quad \forall o
$$

then we also have

$$
\phi^{o}\left(\Pi^{o}(x, \omega), \omega\right)=\phi^{c}(\Pi(\omega), \omega)=\frac{\partial \rho}{\partial \nu(\omega)}[Q(x, \omega)] .
$$

and (58) would be a true equilibrium model with risk averse consumers and generators having the same stochastic discount factor.

Financial products can in principle help achieve equality (65) (Ralph and Smeers, 2010) even if generators are not identical up to a scaling factor. Suppose a market that trades bonds paying coupons indexed on electricity and fuel prices. The owner of a plant could hedge its revenue by a portfolio of these bonds. The consumer could also hedge its electricity payment $\Pi(\omega)$ with these bonds. The difficulty of this reasoning is that it does not apply to idiosyncratic risk that, by definition, cannot be traded. Stating model (57) as an equilibrium model may thus be an interesting counterfactual but it is only an approximation that holds if idiosyncratic risk is not too important.

The same reasoning applies to the Variable Demand Model. Total welfare is the sum of consumer and producer surplus, a relation that we write as

$$
\begin{array}{r}
-M S T W(x, \omega)=\sum_{o \in O} \sum_{\ell \in L} \tau(\ell)\left[\sum_{k \in K} p(\ell, \omega)-c(k, \omega)\right] y^{o}(k, \ell, \omega) \\
+\left[\int_{0}^{d(\ell, \omega)} p(\xi, \ell, \omega) d \xi-p(\ell, \omega) d(\ell, \omega)\right] .
\end{array}
$$

or $\operatorname{MTSW}(x, \omega)=-\sum_{o} \Pi^{o}(x, \omega)-\Pi^{c}(\omega)$ where $\Pi^{o}(x, \omega)$ and $\Pi^{c}(\omega)$ are respectively generator $o$ and consumer $c$ surplus. We can therefore interpret the variable demand version of (58) as an equilibrium model if we can guarantee

$$
\phi^{o}\left(\Pi^{o}(x, \omega), \omega\right)=\phi^{c}\left(\Pi^{c}(\omega), \omega\right)=\frac{\partial \rho}{\partial \nu(\omega)}(M S T W(x, \omega)) .
$$


Equality will hold for all generators if they have the same generation structure (again up to a scaling factor $)$. But equality between $\phi^{o}\left(\Pi^{o}(x, \omega), \omega\right)$ and $\phi^{c}\left(\Pi^{c}(\omega), \omega\right)$ can only hold if consumers and generators can trade idiosyncratic risk. As before, this is not possible by definition.

In order to conclude the discussion consider an alternative market structure where each generator specializes in one technology. The set $O$ of generators $o$ then coincides with the set $K$ of technologies $k$. By construction, we can no longer attempt to make the stochastic discount factors of the different generators equal. A financial market could still entail that equality if idiosyncratic risk could be traded between generators and the consumer. But this is again impossible by definition of the idiosyncratic risk. In conclusion we can retain model (59) completed by the short run equilibrium conditions (6) to (9) or its Variable Demand Model counterpart as an ideal counterfactual but need to consider alternative formulations that better account for the nature of idiosyncratic risk. The following presents different models that one can think of. They are all stated in the Fixed Demand Model form but can easily be adapted to the Variable Demand Model. The two first models have the same short term market submodel (69) to (72); the third model has a slightly modified version that will be presented in due course.

$$
\begin{gathered}
0 \leq x(k)-y(k ; \ell, \omega) \perp \mu(k ; \ell, \omega) \geq 0 \\
0 \leq \sum_{k \in K} y(k ; \ell, \omega)+z(\ell, \omega)-d(\ell, \omega) \perp \pi(\ell, \omega) \geq 0 \\
0 \leq c(k ; \omega)+\mu(k ; \ell, \omega)-\pi(\ell, \omega) \perp y(k ; \ell, \omega) \geq 0 \\
0 \leq P C(\omega)-\pi(\ell, \omega) \perp z(\ell, \omega) \geq 0
\end{gathered}
$$

\subsubsection{Perfect risk trading or perfectly diversified plant portfolios}

Whatever the shortcomings of the optimization model (57) for representing an equilibrium it remains a useful counterfactual because it represents an ideal situation of a complete market where idiosyncratic risk could be traded, for instance through special insurance contracts. Also, because it is a convex optimization problem, it is easy to compute. Note that it may be unbounded (and hence not amenable to an equilibrium interpretation) when $m^{*}(\omega)$ has negative components. We simply restate the investment part (which is similar to the criterion of equation (53) but not with a risk adjustment on welfare instead of costs) of the model here without further discussion.

$$
0 \leq I(K)-R^{f} \frac{\partial \rho(Q(x, \omega), \omega)}{\partial \nu(\omega)} \frac{\partial Q(x, \omega)}{\partial x} \perp x(K) \geq 0
$$


that is rewriten

$$
0 \leq I(K)-R^{f} E_{p}(\phi(x, \omega) \times \mu(x, K, \omega)) \perp x(K) \geq 0 .
$$

\subsubsection{The project finance approach}

The project finance approach is a second counterfactual where each plant is valued on the basis of its own merit. Consider now a market where each agent invests in a particular technology. Let $\rho^{k}$ be the valuation function of agent $k$. The investment criterion leads to a formulation where each investor $k$ solves the following profit maximisation problem

$$
\min _{x(k) \geq 0}\left[I(k)-R^{f} \rho^{k}[\mu(x, k, \omega)]\right] x(k)
$$

where $\mu(x, k, \omega)$ retains its interpretation of marginal value of a unit capacity of plant $k$ in the short run equilibrium when the system portfolio is $x$. The corresponding investment condition for plant of type $k$ is then

$$
0 \leq I(k)-R^{f} E_{p} \phi^{k}(x, \omega) \mu(x, k, \omega) \perp x(k) \geq 0
$$

where $\phi^{k}(x, \omega)=m^{\star}(\omega)+m^{k}\left(\sum_{\ell \in L} \tau(\ell) \mu(x, k, \ell, \omega), \omega\right)$. This condition applies to all plants $k$ each one being written with plant $k$ specific stochastic discount factor $\phi^{k}$ (compare with Section 4 where a simple stochastic discount factor applies to all plants). This problem can easily be converted into a variational inequality problem if, as is often the case there are constraints on investment possibilities. Each mapping $-E_{p} \phi^{k}(x, \omega) \mu(x, k, \omega)$ is monotone if $m^{\star}(\omega)$ is non negative. Consider now the global investment criterion

$$
0 \leq I(K)-F(x) \perp x(K) \geq 0
$$

where $F^{k}(x)=-R^{f} E_{p} \phi^{k}(x, \omega) \mu(x, k, \omega)$. Even though each $F^{k}(x)$ is monotone, the mapping $F(x)$ does not necessarily satisfying this property.

\subsubsection{Diversified portfolio models}

We now come back to the model with different generators $o$ that each invest in and operate a mix of generating capacities. For the sake of realism we here complicate the problem and suppose that each operator has an existing generation fleet $\bar{x}^{o}(k)$ and is considering investing $x^{o}(k)$. We define

$$
\begin{aligned}
& \bar{x}(k)=\sum_{o \in O} \bar{x}^{o}(k) \\
& x(k)=\sum_{o \in O} x^{o}(k)
\end{aligned}
$$


and modify the equilibrium conditions of the short run market in order to account for existing capacities. These become

$$
\begin{gathered}
0 \leq \bar{x}(k)+x(k)-y(k ; \ell, \omega) \perp \mu(k ; \ell, \omega) \geq 0 \\
0 \leq \sum_{k \in K} y(k ; \ell, \omega)+z(\ell, \omega)-d(\ell, \omega) \perp \pi(\ell, \omega) \geq 0 \\
0 \leq c(k ; \omega)+\mu(k ; \ell, \omega)-\pi(\ell, \omega) \perp y(k ; \ell, \omega) \geq 0 \\
0 \leq P C(\omega)-\pi(\ell, \omega) \perp z(\ell, \omega) \geq 0
\end{gathered}
$$

The profit of generator $o$ in state of world $\omega$ is then

$$
\Pi^{o}(\bar{x}+x ; \omega) \equiv \sum_{k}\left[\bar{x}^{o}(k)+x^{o}(k)\right] \mu[\bar{x}(k)+x(k) ; \omega]
$$

The investment criterion needs now be rewritten for each generator $o$

$$
0 \leq I(k)-R^{f} E_{p}\left[\phi^{o}\left[\Pi^{o}(\bar{x}+x ; \omega) ; \omega\right] \mu[\bar{x}(k)+x(k) ; \omega] \perp x^{o}(k) \geq 0 .\right.
$$

This criterion applies to the set of plants and can thus be rewritten as

$$
0 \leq I(K)-F^{0}(x) \perp x^{0}(K) \geq 0
$$

where $F^{0}(x)=R^{f} E_{p}\left[\phi^{0}\left[\pi^{0}(\bar{x}+x ; \omega) \mu[\bar{x}(K)+x(K) ; \omega]\right.\right.$. The criterion must be stated for each generator $o$. As for the project finance model, this mapping is not monotone in general. This problem can easily be converted into a variational inequality problem if, as is often the case there are constraints on investment possibilities.

\subsubsection{Comment}

The loss of monotonicity in the "project finance approach" and the "diversified portfolio models" has an economic interpretation. It is related to the need to value idiosyncratic risks that by definition cannot be traded. This brings us into the domain of incomplete markets. We shall come back to this phenomenon in further papers.

\section{Numerical Illustration}

\subsection{The set-up}

In this section we illustrate the impact of the investment criteria discussed in the main text on a simple but realistic example that exhibits different relevant features. Very much like the rest of the paper, the 
example illustrates a skeleton of real models. We first present the data for the deterministic model that we then extend to a stochastic version. Consider a two stage problem: one invests in different types of capacities in stage 0 and operates them in stage 1. Following Joskow (2007), our benchmark example is a three technology problem involving coal, combined cycle gas turbine (CCGT) and open cycle gas turbine (OCGT). Each equipment type is characterized by its annual investment and fixed operating costs and a $\mathrm{CO}_{2}$ emission factor. Because risk premia are endogenous in the stochastic equilibrium model, investment costs are meant to be annualized from overnight construction costs at the risk free rate (see Table 1).

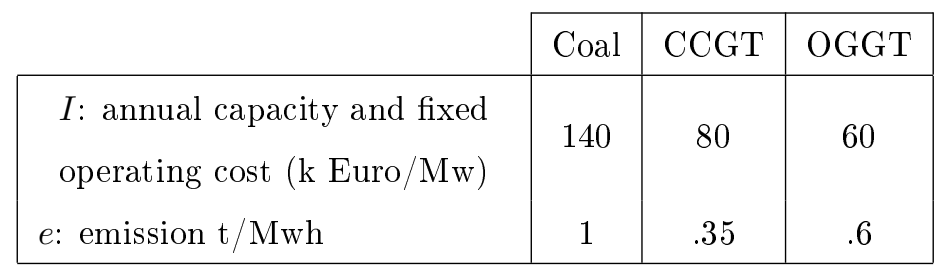

Table 1: Fixed annual cost and emission in a three technology world

All costs are assumed linear in capacity or operations levels implying that we neglect economies of scale in generation. This assumption is common in capacity expansion models. Emission factors for each plant are in tons of $\mathrm{CO}_{2}$ per MWh. These figures are stylized views on costs and emission factors found in the European industry at the time of this writing. They do not correspond to particular projects but are realistic. The operating costs will be derived from fuel prices.

The deterministic model supposes one fuel price scenario and one scenario for CO2 prices. We assume a simple price cap throughout the paper. These are shown in Table 2.

The load duration curve is segmented in 6 demand blocks in order to keep the model simple, while still guaranteeing sufficient detail for arriving at meaningful results. Table 3 gives the relevant figures and units.

\subsection{Introducing risk (Section 1.2)}

The deterministic model only involves a single $\omega \in \Omega$. The stochastic model resorts to three probability spaces (Load, fuel and $\mathrm{CO} 2$ ) and hence three probability measures. We generate three possible realisations for each risk factor multiplying the hourly load level, variable operating costs and $\mathrm{CO} 2$ prices with the 


\begin{tabular}{|c|c|c|c|}
\hline & Coal & CCGT & OGGT \\
\hline $\begin{array}{l}c(k) \text { : fuel and variable } \\
\text { operating cost ( Euro/Mwh) }\end{array}$ & 25 & 45 & 80 \\
\hline CO2: price (Euro/ton) & 20 & & \\
\hline$P C:$ price cap (Euro/Mwh) & 300 & & \\
\hline
\end{tabular}

Table 2: Variable cost, price cap and CO2 price

\begin{tabular}{|c|c|c|c|c|c|c|}
\hline \multicolumn{7}{|c|}{ power level and utilisation } \\
\hline$d:$ MW & 86000 & 83000 & 80000 & 60000 & 40000 & 20000 \\
$\tau:$ duration (1000 hours) & .01 & .04 & .31 & 4.4 & 3 & 1 \\
\hline
\end{tabular}

Table 3: Reference load duration curve and its decomposition in time segments

factors listed in Table 4. Combining the three values we end up with 27 scenarios that are assumed equally probable.

\subsection{Alternative equilibrium models (Section 2): $\mathrm{CO}_{2}$ cap and free allocation}

In order to illustrate the flexibility of the equilibrium framework we also consider slightly more complicated versions of the simple capacity expansion model used throughout the paper where we include a simple representation of the EU-ETS (Emission (of $\mathrm{CO}_{2}$ ) Trading Scheme, Ehrenmann and Smeers, 2010). Assuming then that the $\mathrm{CO} 2$ price is determined in a cap and trade system we explicitly model the cap. Starting from the emissions obtained in the deterministic base case which are found equal to $219 \mathrm{mt}$, we

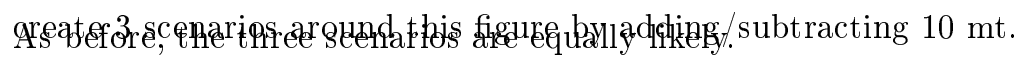

We complicate this EU-ETS model and depart from an optimization model (see Ehrenmann and Smeers, 2010) for a discussion of that extension) by considering the case where free allowances are 


\begin{tabular}{|l|c|c|c|}
\hline & low & central & high \\
\hline Load & $90 \%$ & $100 \%$ & $110 \%$ \\
Fuel & $70 \%$ & $100 \%$ & $130 \%$ \\
CO2 price & $50 \%$ & $100 \%$ & $150 \%$ \\
\hline
\end{tabular}

Table 4: Scenario generation

\begin{tabular}{|c|c|c|c|}
\hline & low & central & high \\
\hline CO2 cap & $209 \mathrm{mt}$ & $219 \mathrm{mt}$ & $229 \mathrm{mt}$ \\
\hline
\end{tabular}

Table 5: Scenario generation

granted to investors. These are usually linked to the expected running hours and to a share of expected emissions covered by free allocation. We use expected running hours of $8000 \mathrm{~h}$ for Coal, $6000 \mathrm{~h} \mathrm{CCGT}$ and of $1000 \mathrm{~h}$ for GT and assume a $20 \%$ coverage. The free allocation rule is then: free allocation= expected running hours $\mathrm{x}$ emission rate $\mathrm{x}$ coverage.

\subsection{Alternative equilibrium models (Section 2): Elastic Demand}

We consider two cases of elastic demand. The first one is the standard situation where one assumes that there is no cross price elasticity: demand in one time segment only depends on the price in that time segment. The demand function is affine and calibrated as follows: we use a reference price equal to the Long Run Marginal Cost of Coal (LRMC) at $8000 \mathrm{~h}$ for the two lowest demand blocks, the LRMC of CCGT at $6000 \mathrm{~h}$ for the intermediate demand and the LRMC of a GT at $1000 \mathrm{~h}$. These are computed at the fuel and $\mathrm{CO} 2$ prices of the deterministic case.

For a given elasticity we calculate the electricity consumption in node $i$ as a function of the price :

$$
Q_{i}=\alpha_{i}-\beta_{i}\left(\text { price }_{i}\right) .
$$




\begin{tabular}{|c|c|c|c|c|c|c|}
\hline \multicolumn{7}{|c|}{ power level and utilisation } \\
\hline$d:$ MW & 86000 & 83000 & 80000 & 60000 & 40000 & 20000 \\
Reference price & 152 & 152 & 65.3 & 65.3 & 62.5 & 62.5 \\
Elasticity & .5 & .5 & .5 & .5 & .5 & .5 \\
\hline
\end{tabular}

Table 6: Reference load and price

We calibrate the coefficients $\alpha_{i}$ and $\beta_{i}$ by writing

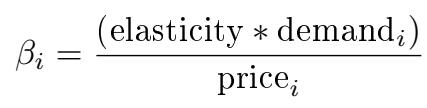

and

$$
\alpha_{i}=\operatorname{demand}_{i}+\operatorname{price}_{i} * \beta_{i} .
$$

The second case deals with the much more novel situation where one supposes that developments of the smart grid type allow for cross substitutions between time segments. We modify the demand function into

$$
Q_{i}=\alpha_{i}-\sum_{j} \beta_{i j}\left(\text { price }_{j}\right),
$$

which makes demand in some time segment dependent on the electricity price in other time segments. We set the cross elasticities so that the off-peak demand increases if peak prices are very high, thereby reproducing the storage effect intended in smart grid developments.

\begin{tabular}{|l|r|}
\hline$\beta_{61}$ & -0.1 \\
$\beta_{62}$ & -0.1 \\
$\beta_{51}$ & -0.1 \\
$\beta_{52}$ & -0.1 \\
\hline
\end{tabular}

Table 7: Elasticities 


\subsection{Systematic risk and linear discount factor (Section 4)}

As explained in the text, the pricing of risk correlated to market risk requires inserting information on market returns in the scenarios. In order to simplify matters we assume that the market return is highly correlated with electricity consumption, fuel prices and, to a smaller extent with load. The results together with the impact on the stochastic discount factor are given in Table 8.

\subsection{Idiosyncratic risk and nonlinear discount factor (Section 5)}

The pricing of idiosyncratic requires a risk function. We use the CVAR, which is now becoming standard and is quite amenable to computation. We account for risk aversion (in this case a prudent evaluation of the payoffs) by ignoring the highest $5 \%$ realizations. In order not to mix effects we run this case without pricing market risks (setting $m^{\star}(\omega)$ to zero). All the risk is thus assumed idiosyncratic.

\begin{tabular}{|l|l|l|c|c|}
\hline Fuel & Load & $\mathrm{CO}_{2}$ & Market return & $m(\omega)$ \\
\hline Low & Low & Low & .55 & 1.421 \\
Low & Low & Medium & .6 & 1.380 \\
Low & Low & High & .65 & 1.338 \\
Low & Medium & Low & 0.95 & 1.087 \\
Low & Medium & Medium & 1.0 & 1.045 \\
Low & High & Low & 1.05 & 1.003 \\
Low & High & Medium & 1.35 & 0.753 \\
Low & High & High & 1.4 & 0.711 \\
Medium & Low & Low & 0.65 & 0.669 \\
Medium & Low & Medium & 0.7 & 1.338 \\
Medium & Low & High & 0.75 & 1.296 \\
Medium & Medium & Low & 1.05 & 1.003 \\
Medium & Medium & Medium & 1.1 & 0.962 \\
Medium & Medium & High & 1.15 & 0.920 \\
Medium & High & Low & 1.45 & 0.669 \\
Medium & High & Medium & 1.5 & 0.627 \\
Medium & High & High & 1.55 & 0.585 \\
High & Low & Low & 0.75 & 1.254 \\
High & Low & Medium & 0.8 & 1.212 \\
High & Low & High & 0.85 & 1.171 \\
High & Medium & Low & 1.15 & 0.920 \\
High & Medium & Medium & 1.2 & 0.878 \\
High & Medium & High & 1.25 & 0.836 \\
High & High & Low & 1.55 & 0.585 \\
High & High & Medium & 1.6 & 0.543 \\
High & High & High & 1.65 & 0.502 \\
\hline
\end{tabular}

Table 8: Market return and linear stochastic discount factor 


\subsection{Simulation results}

The following table summarizes the computational results; we only report the investments in the different types of plants, the total capacity and the shortfall (see Joskow , 2007 and the discussion of the "missing money" for an explanation of the shortfall) and the average baseload price:

\begin{tabular}{|l|c|c|c|c|c|c|}
\cline { 2 - 6 } \multicolumn{1}{c|}{} & Coal & CCGT & OCGT & Total & Max Shortfall & Average Baseload Price \\
\hline Deterministic - (Section 1.2) & 20000 & 40000 & 20000 & 80000 & 6000 & 60.98 \\
Stochastic - (Section 1.3) & 20000 & 46000 & 6000 & 72000 & 22.6 & 60.70 \\
Elastic demand - (Section 2.1) & 20340 & 40780 & 0 & 61120 & 0 & 60.89 \\
Elastic Dmand DSM - (Section 2.3) & 24120 & 37690 & 0 & 61810 & 0 & 60.91 \\
CO2 constraint - (Section 2.3) & 27200 & 38800 & 6000 & 72000 & 22600 & 59.85 \\
Free Allocation - (Section 2.3.) & 28890 & 43110 & 0 & 72000 & 22600 & 60.25 \\
Linear discount factor - (Section 4) & 12000 & 54000 & 6000 & 72000 & 22600 & 61.71 \\
CVAR - (Section 5) & 18000 & 48000 & 6000 & 72000 & 22600 & 61.16 \\
\hline
\end{tabular}

Table 9: Computational results

The table provides a direct quantification of important effects. Moving from a deterministic to a stochastic environment reinforces the partial load capacities (CCGT). Introducing cross time segment substitution (moving from Elastic Demand to Elastic Demand with DMS) implies a shift from peak (OCGT) to base (coal) units. The free allocation of permits (moving from $\mathrm{CO}_{2}$ constraint to Free Allocation) effectively acts as a strong incentive to investments. Finally the introduction of a CVaR shifts investments from high upfront capital expenditure (coal) to lower upfront capital expenses. All these phenomena are expected. The equilibrium model allows one to quantitatively explore them.

\section{Conclusion}

The optimization capacity expansion models of the regulatory period have not yet found their counterpart in the restructured electricity markets. We present different adaptations of these former optimization models to the new competitive environment. Our objective is twofold: the model should be interpretable in terms of market equilibrium in order to fit with the new competition environment; it should also 
account for risk, which is becoming daunting in the industry. We begin with a simple standard two-stage version of a stochastic optimization capacity expansion model that we immediately convert into a parsimonious complementarity representation of the investment problem. The discussion then proceed as follows. Even though we start with an optimization model, we show that the equilibrium formulation can encompass much more general models that do not derive from an optimization problem; this is important in a context where demand resources will play an increasing role. This is also important because of the flurry of new policies affecting the sector and distorting its environment from normal competition conditions. The rest of the paper deals with different treatments of risk encountered in practice. While a pure risk neutral version of the model is straightforward to construct, things become more difficult if one wants to account for risk aversion and how it is treated in corporate finance. CAPM driven evaluations are common in practice and the philosophy of project finance requires to value each plant according to its own risk profile. This is usually done by discounting the cash flows of individual plants at a plant specific risk adjusted discount rate. Because the risk exposure of a plant depends on the development of the generation system, this approach effectively poses a problem of fixed point, a much more complex object than what is usually envisaged in practice. We bypass this difficulty by invoking stochastic discount factors that we directly embed in the equilibrium models. Discounting then takes place at the risk free rate, therefore eliminates difficulties that occur when different plants are affected by different discount rates. The counterpart is the need to compute risk adjusted or deterministic equivalents of cash flows. We discuss two different approaches both based on stochastic discount factors. Linear discount factors come for standard economic theories like the CAPM, the APT or the multitemporal consumer theory. They price what is commonly known as "systematic risk". They fully accommodate the requirements of project finance that each plant should be valued on the basis of its own risk exposure. But they limit this valuation to the risk priced by the market, something which is often, but not always accepted in practice. Difficulties arise when one insists on accounting for idiosyncratic risk. We do this by resorting to risk functions that we embed in the equilibrium context. The extension raises interesting questions: while the insertion of risk functions in a capacity expansion optimization model maintains convexity properties, this is not so in equilibrium models when we describe idiosyncratic risk by risk functions. The models can still be written without difficulties. But they loose their convexity properties. This paper does not discuss the interpretation of this loss of convexity but it suggests that it is deep: because idiosyncratic risk cannot be traded, the market becomes incomplete a phenomenon that is revealed by the loss the mathematical properties that guarantee existence and uniqueness of solution (here equilibrium). A numerical illustration illustrates the different features treated in the paper. 


\section{Appendix 1}

\section{The optimization model as a perfect competition equilibrium model}

Suppose a set of generators, and a set of time segments. A perfect competition equilibrium is a vector of electricity prices (one price per time segment) and a vector of investments (one per type of capacity) and generation levels (one per type of capacity and time segment), such that investments and operations maximize generators' profits at prevailing prices and total generation matches demand in each time segment.

More explicitly in the single stage view of the equilibrium, a perfect competition equilibrium consists of a set of electricity prices $\pi(\ell)$ and a set in investment and operations levels $x(k)$ and $y(k, \ell)$, such that $x(k)$ and $y(k, \ell)$ maximize the profit of generator of capacity $k$ at prices $\pi(\ell)$ and the sum of the $y(k, \ell)$ over all $k$ matches demand $d(\ell)$ in time segment $\ell$.

Alternatively a two stage view of the problem considers a set of investors in generation capacities that build/buy capacities and lease them (tolling agreement) to a set of operators of generation capacities that rent these capacities and sell electricity in a set of time segments. In this set up, a perfect competition equilibrium is a vector of electricity prices (one per time segment), a vector of capacity prices (one per time segment), a vector of investment levels (one per type of plant), and a vector of operations levels (one per type of plant and time segment), such that investment levels maximize investors' profits at prevailing capacity prices and operations levels maximize operators' profits at prevailing electricity and capacity prices.

More explicitly, in the the operators' model, a perfect competition equilibrium in the second stage consists of a set of electricity prices $\pi(\ell)$, a set of rental capacity prices $\mu(k, \ell)$, a set of capacities $x(k)$ of plant $k$, and a set of operations level $y(k, \ell)$, such that the operator of technology maximizes its profit by renting $x(k)$ of capacity $k$ at price $\mu(k, \ell)$ and selling $y(k, \ell)$ with that capacity at price $\pi(\ell)$ and the sum of the $y(k, \ell)$ over all $k$ matches demand $d(\ell)$ in time segment $\ell$. Similarly, in the investor's and overall models, a perfect competition equilibrium in the first stage consists of a set of capacity rental 
prices $\mu(k, \ell)$, a set of investments in capacity $x(k)$ such that $x(k)$ maximizes the profit of investors in technology $k$ when rental price is $\mu(k, \ell)$. The two stage perfect competition equilibrium is a set of electricity price $\pi(\ell)$ and rental price $\mu(k, \ell)$, investments $x(k)$ and operations levels $y(k, \ell)$ that form a perfect competition equilibrium in both the first and second stages.

\section{Proposition : relation between the optimization and the perfect competition equilibrium problems}

The two definitions of the perfect competition equilibrium are equivalent in the sense that a perfect competition equilibrium of one type is also a perfect competition equilibrium of the other type. The solution of the optimization problem is a perfect competition equilibrium and conversely.

Proof. Verify that the complementarity conditions of the three problems are identical.

\section{Appendix 2: Properties of $\mu(x, K)$}

\section{1. $\mu(x, K)$ is a subgradient of $Q(x)$}

Rewrite problem (1) as

$$
Q(x)=\sum_{\ell \in L} \tau(\ell) Q(x, \ell)
$$

where

$$
\begin{aligned}
& Q(x, \ell)=\min _{y(k, \ell), z(\ell)} \sum_{k \in K}[c(k) y(k, \ell)+\mathrm{PC} z(\ell)] \\
& \text { s.t. } \quad 0 \leq x(k)-y(k, \ell) \\
& 0 \leq \sum_{k \in K} y(k, \ell)+z(\ell)-d(\ell) \\
& \mu(k, \ell) \\
& \leq y(k, \ell) \\
& \pi(\ell)
\end{aligned}
$$

One knows that

$$
\mu(K, \ell) \in \partial_{x} Q(x, \ell)
$$

and hence since

$$
\begin{aligned}
Q(x) & =\sum_{\ell \in L} \tau(\ell) Q(x, \ell) \\
\mu(K) & =\sum_{\ell} \tau(\ell) \mu(K, \ell) \in \partial Q(x)
\end{aligned}
$$




\section{2. $\mu(x ; K)$ is multivalued}

Consider a time segment $\bar{\ell}$ and a set of capacities $x(k)$ such that

$$
\begin{array}{ll}
0=x(k)-y(k, \bar{\ell}) & k=1, \cdots, k^{*} \\
0=y(k, \bar{\ell}) & k>k^{*} \\
\sum_{k} y(k, \bar{\ell})=d(\bar{\ell}) . &
\end{array}
$$

One can check that

$$
\begin{array}{ll}
\pi(\bar{\ell})=c\left(k^{*}-1\right) & \\
\mu(k, \bar{\ell})=\pi(\bar{\ell})-c(k) & k \leq k^{*}-1 \\
\mu(k, \bar{\ell})=0 & k \geq k^{*}
\end{array}
$$

is a vector of dual variables satisfying the short term model. Alternatively, one also verifies that

$$
\begin{array}{ll}
\pi(\bar{\ell})=c\left(k^{*}\right) & \\
\mu(k, \bar{\ell})=\pi(\bar{\ell})-c(k) & k \leq k^{*} \\
\mu(k, \bar{\ell})=0 & k>k^{*}
\end{array}
$$

is also a vector of dual variables satisfying the short term model.

\section{3. $\mu(x, K)$ is a gradient of $\operatorname{MSTW}(x, \omega)$}

Rewrite problem (24) to (27)

$$
\operatorname{MSTW}(x, \omega)=\sum_{\ell \in L} \tau(\ell) \operatorname{MSTW}(x, \omega, \ell)
$$

where

$$
\begin{array}{rlr}
\operatorname{MSTW}(x, \omega, \ell) & =\min _{y(k, \ell)}\left[\sum_{k \in K} c(k, \omega) y(k, \ell, \omega)-\int_{0}^{d(\ell, \omega)} p(\xi, \ell, \omega) d \xi\right] \\
\text { s.t. } & 0 \leq x(k)-y(k, \ell, \omega) & \mu(k, \ell) \\
0 & \leq \sum_{k \in K} y(k, \ell, \omega)-d(\ell, \omega) & \pi(\ell) \\
0 & \leq y(k, \ell, \omega) .
\end{array}
$$

The function is differentiable for any point $x(K)$ where the short term complementarity conditions are strict, that is such that $0=x(k)-y(k, \ell)$ implies $y(k+1, \ell)>0$. Consider a point where this condition is not satisfied, that is,

$$
\begin{aligned}
& y\left(k^{*}, \ell, \omega\right)=0<x\left(k^{*}\right) \\
& 0<y\left(k^{*}-1, \ell, \omega\right)=x\left(k^{*}-1\right) .
\end{aligned}
$$


Because $d(\ell, \omega)>0$ one has

$$
\pi(\ell, \omega)=p(d(\ell, \omega)
$$

which is uniquely determined and hence

$$
\mu\left(k^{*}-1, \ell, \omega\right)=\pi(\ell, \omega)-c\left(k^{*}-1, \omega\right)
$$

is also uniquely determined.

Last, $\mu\left(k^{*}, \ell, \omega\right)=0$ since

$$
x\left(k^{*}\right)-y\left(k^{*}, \ell, \omega\right)>0 .
$$

The subdifferential boils down to a single point. Because the subdifferential is upper semicontinuous, this implies that it is continuous.

\section{Appendix 3: Definition of monotonicity}

Let $\mu(x)$ be a mapping from $R^{n}$ into $R^{n} \cdot \mu(x)$ is monotone if

$$
[\mu(x)-\mu(y)]^{T}(x-y) \geq 0 \text { for all } x \text { and } y .
$$

The definition extends to point to set mapping as follows. Let $\mu(x)$ be a point to set mapping from $R^{n}$ to $R^{n} \cdot \mu(x)$ is monotone if for any $x, y$, there exists

$$
\mu^{x} \in \mu(x) ; \quad \mu^{y} \in \mu(y)
$$

such that

$$
\left(\mu^{x}-\mu^{y}\right)^{T}(x-y) \geq 0 .
$$

\section{Appendix 4: Definition of coherent risk function}

The following definitions are taken from Shapiro et al. (2009).

- A. Let $\mathcal{Z}$ be a linear space of random outcome $Z(\omega) . \rho(\mathcal{Z})$ satisfies the following axioms.

- A1. Convexity. $\rho\left(t Z+(1-t) Z^{\prime}\right) \leq t \rho(Z)+(1-t) \rho\left(Z^{\prime}\right)$ for all $t \in[0,1]$ and all $Z, Z^{\prime} \in \mathcal{Z}$

- A2. Monotonicity. If $Z, Z^{\prime} \in \mathcal{Z}$ and $Z \geq Z^{\prime}$ the $\rho(Z) \geq \rho\left(Z^{\prime}\right)$.

- A3. Translation equivariance if $a \in R$ and $Z \in \mathcal{Z}$ then $\rho(Z+a)=\rho(Z)+a$ 
- A4. Positive homogeneity. If $t>0$ and $Z \in \mathcal{Z}$ then $\rho(t Z)=t \rho(Z)$.

The move from a risk function to a valuation function obtains by replacing the convexity axiom A1 by a concavity axiom A'1.

- A'1. Concavity. $\rho\left(t Z+(1-t) Z^{\prime}\right) \geq t \rho(Z)+(1-t) \rho\left(Z^{\prime}\right)$ for all $t \in[0,1]$ and all $Z, Z^{\prime} \in \mathcal{Z}$.

\section{References}

[1] Aghassi, M., Bertsimas, D. and G. Perakis (2006). Solving asymmetric variational inequalities via convex optimization. Operations Research Letters, 34, 481-490.

[2] Ahn, B.-H. and W.W. Hogan (1982). On convergence of the PIES algorithm for computing equilibria. Operations Research, 30(2), 282-300.

[3] Armitage, S. (2005). The Cost of Capital, Intermediate Theory. Cambridge University Press, Cambridge.

[4] Artzner, P., Delbaen, F., Eber, J.-M. and D. Heath (1999). Coherent measures of risk. Mathematical Finance, 9(3), 203-228.

[5] Artzner, P., Delbaen, F., Eber, J.-M. and H. Ku (2007). Coherent multiperiod risk adjsuted values and Bellman's principle. Annals of Operational Research, 152, 5-22.

[6] Cochrane, J.H. (2005). Asset Pricing. Princeton University Press, Princeton and Oxford.

[7] Eichhorn, A., Heitsch, H. and W. Römisch (2010). Stochastic optimiation of electricity portfolios: scenario tree modeling and risk management. In P.M. Pardalos, M.V.F. Pereira, S. Rebennack and N.A. Iliadis, Handbook of Power Systems. Berlin, Springer.

[8] Ehrenmann, A. and Y. Smeers (2009). Risk adjusted discounted cash flows in capacity expansion models. Presentation at the MPS 2009, Chicago. Slides available, paper in preparation.

[9] Ehrenmann, A. and Y. Smeers (2010). Generation capacity expansion in a risky environment: a stochastic equilibrium analysis. Accepted for publication in Operations Research.

[10] Fama, E. (1977). Risk-adjsuted discount rates and capital budgeting under uncertainty. Journal of Financial Economics, 5(1), 3-24. 
[11] Facchinei, F. and J.-S. Pang (2003). Finite-Dimensional Variational Inequalities and Complementarity Problems. New-York, Springer.

[12] Fama, E. (1977). Risk-adjsuted discount rates and capital budgeting under uncertainty. Journal of Financial Economics, 5(1), 3-24.

[13] Gabriel, S.A., Kydes, A.S. and P. Whitman (2001). The national energy modeling system: a largescale energy-economic equilibrium model. Operations Research, 49(1), 14-25.

[14] Grahan, J. and C. Harvey (2001). The theory and practice of corporate finance: Evidence form the field. Journal of Financial Economics, 60(2-3), 187-243.

[15] Gürkan, G., Özge, A.Y. and S.M. Robinson (1999). Sample-path solutions of stochastic variational inequalities. Mathematical Programming, 84, 313-333.

[16] Gürkan, G., Özge, A.Y. and Y. Smeers (2009).Capacity investment in electricity markets: perfect competition. Discussion Paper, Department of Econometrics and Operations Research, Universiteit Tilburg, 2009.

[17] Harker, P.T. and J.-S. Pang (1990). Finite-dimensional variational inequality and nonlinear complementarity problems: a survey of theory, algorithms and applications. Mathematical Programming, Series $A$ and $B, 48(2), 161-220$.

[18] Haurie, A., Legrand, J., Smeers, Y. and G. Zaccour (1988). A stochastic dynamic Nash Cournot model for the European Gas market. Les Cahiers du GERAD, C-87-24, Octobre 1988.

[19] Hogan, W.W., Gribik, P.R. and S.L. Pope (2007). Market-clearing electricity prices and energy uplift. Available from Harvard Electricity Policy Group (HEPG website), Harvard University, December 2007.

[20] Joskow, P.L. (2007). Competitive electricity markets and investments in new generation capacity. In Helm, D. (ed.), The New Energy Paradigm, Oxford University Press, Oxford.

[21] Kuhn, S. and R. Schultz (2009). Risk neutral and risk averse power optimization in electricity networks with dispersed generation. Mathematical Methods of Operations Research, 69(2), 353-367.

[22] Lin, G.-H. and M. Fukushima (2009). Stochastic equilibrium problems and stochastic mathematical programs with equilibrium constraints. A survey. Technical Report, Department of Applied Mathematics and Physics, Kyoto University, March 2009. 
[23] Morlat, G. and F. Bessière (1971). Vingt cinq ans d'Economie electrique. Paris, Dunod.

[24] Ralph, D. and Y. Smeers (2010). Risk averse structure equilibria: a perfectly competitive two-stage market in capacity, financial securities and production. In preparation.

[25] Römisch, W. and S. Vigerske (2010). Recent progress in two-stage mixed-integer stochastic programming with applications in power production planning. In P.M. Pardalos, M.V.F. Pereira, S. Rebennack and N.A. Iliadis, Handbook of Power Systems. Berlin, pringer.

[26] Shapiro, A. (2009). On a time consistency concept in risk averse multistage stochastic programming. Operations Research Letters, 37, 143-147, 2009.

[27] Shapiro, A., Dentcheva, D. and A. Ruszczynski (2009). Lectures on Stochastic Programming: Modeling and Theory, MPS/SIAM Series on Optimization 9, Philadelphia, SIAM-Society for Industrial and Applied Mathematics.

[28] Wu, Y.J. and J.D. Fuller (1996). An algorithm for the multiperiod market equilibrium model with geometric distributed lag demand. Operations Research, 44(6), 1002-1012. 